\title{
HLA-DR expression in clinical-grade bone marrow-derived multipotent mesenchymal stromal cells: a two-site study
}

\author{
Marta Grau-Vorster ${ }^{1,2 \dagger}$, Anita Laitinen ${ }^{3 \dagger}$, Johanna Nystedt ${ }^{3 *}$ and Joaquim Vives ${ }^{1,4,5^{*}}$ (D)
}

\begin{abstract}
Background: Contrary to the minimal criteria proposed by the International Society for Cell and Gene Therapy for defining multipotent mesenchymal stromal cells (MSC), human leukocyte antigen (HLA)-DR expression is largely unpredictable in ex vivo-expanded clinical-grade cultures. Although activation of MSC in culture does not appear to affect their functionality, a large study investigating the impact of HLA-DR expression on cell identity and potency is still missing in the literature.

Methods: A retrospective analysis of HLA-DR expression in 130 clinical batches of bone marrow (BM)-MSC from two independent Good Manufacturing Practice-compliant production facilities was performed in order to identify the consequences on critical quality attributes as well as potential activation cues and dynamics of MSC activation in culture.
\end{abstract}

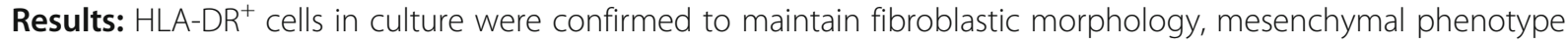
identity, multipotency in vitro, and immunomodulatory capacity. Interestingly, the use of either human sera or platelet lysate supplements resulted in similar results.

Conclusions: HLA-DR expression should be considered informative rather than as a criterion to define MSC. Further work is still required to understand the impact of HLA-DR expression in the context of product specifications on BM-MSC qualities for clinical use in specific indications.

Keywords: Multipotent mesenchymal stromal cell, Potency, Identity, HLA-DR, Cellular therapy, Cell culture, Quality compliance

\section{Introduction}

Multipotent mesenchymal stromal cells (MSCs) can be easily derived from bone marrow (BM) aspirates and further expanded ex vivo up to sufficient numbers for clinical use [1,2]. Indeed, several clinical applications of MSC are currently being explored in the fields of transplantation and regenerative medicine, with a couple of products already holding marketing approval [3-5]. In addition to their potential to become specialized bone, cartilage, and fat cells, the use of MSC is of particular

\footnotetext{
* Correspondence: johanna.nystedt@gmail.com; jvives@bst.cat

${ }^{+}$Marta Grau-Vorster and Anita Laitinen contributed equally to this work

${ }^{3}$ Finnish Red Cross Blood Service, Advanced Cell Therapy Centre, Kivihaantie

7, FIN-00310 Helsinki, Finland

'Servei de Teràpia Cel.lular, Banc de Sang i Teixits, Edifici Dr. Frederic Duran i

Jordà, Passeig Taulat, 116, 08005 Barcelona, Spain

Full list of author information is available at the end of the article
}

interest in the management of graft rejection and/or inflammatory conditions, due to their capacity to modulate the biological response of other cell types involved in these processes [6]. In view of their increasing popularity of MSC as a candidate drug for use in the treatment of a range of pathologies, the International Society for Cell and Gene Therapy (ISCT) established minimal criteria to define MSC identity in an attempt to standardize the product specifications and methods for characterization of MSC regardless of their origin and derivation protocols [7]. ISCT's criteria are based on MSC's adherence to plastic surfaces, multipotency in vitro, and the expression of a set of cell surface markers (positive for CD73, CD90, and CD105; negative for CD14, CD34, CD45 or CD11b, CD79 $\alpha$ or CD19, and HLA-DR). Intriguingly, BM-MSC do express HLA-DR not only when

(C) The Author(s). 2019 Open Access This article is distributed under the terms of the Creative Commons Attribution 4.0 International License (http://creativecommons.org/licenses/by/4.0/), which permits unrestricted use, distribution, and 
inflammatory molecules such as IFN- $\gamma$ are present but also under normal expansion culture conditions $[8,9]$.

In the present study, we re-analyzed HLA-DR expression in 130 clinical batches of BM-MSC from two independent Good Manufacturing Practice (GMP)-compliant production facilities to study its potential effect on the attributes of MSC and the dynamics of activation in expansion cultures.

\section{Methods}

\section{Cells and cell culture}

In Barcelona, clinical-grade BM-MSC were produced within the context of four clinical trials (2009-01644924, 2010-023998-18, 2013-005025-23, 2010-023999-12) with appropriate donor informed consent and approval from a competent regulatory authority. When needed, cells were further expanded in vitro up to sufficient numbers (always under passage 4) using Dulbecco's modified Eagle's medium (DMEM; Gibco) supplemented with $10 \%$ human serum B (hSerB) containing $2 \mathrm{mM}$ glutamine in T-flasks and CellSTACKS (Corning Incorporated Life Sciences) at $1 \times 10^{3}$ to $3.5 \times 10^{3}$ cells $/ \mathrm{cm}^{2}$ seeding density [10]. All cultures were maintained at $37^{\circ} \mathrm{C}$ and $5 \% \mathrm{CO}_{2}$ in humidified incubators. Cell counting and viability assessment of $\mathrm{BM}$ samples were performed using a Guava EasyCyte Mini (Millipore).

In Helsinki, donor recruitment, eligibility assessment, and bone marrow aspiration protocol were approved by the local ethics committee, and the BM collection procedure was authorized by the Finnish Medicines Agency under a tissue establishment license. MSCs were produced under a national advanced therapy medicinal products (ATMP) hospital exemption license authorized by the Finnish Medicines Agency (Fimea; national ATMP manufacturing licenses \#6322/20.10.01/2011 and \#5103/20.30.01/2013). MSC were derived from BM aspirates as reported elsewhere [11]. Briefly, primary cultures (P0) were initiated by plating isolated $\mathrm{BM}$ mononuclear cells (MNCs) at $4 \times 10^{5}$ cells $/ \mathrm{cm}^{2}$. The MSCs were expanded in a medium consisting of Dulbecco's modified Eagle's medium (DMEM) low glucose (Life Technologies) supplemented with $10 \%$ human Platelet Lysate (hPL) and $40 \mathrm{UI} / \mathrm{mL}$ heparin (Heparin LEO $5000 \mathrm{IE} / \mathrm{KY} / \mathrm{mL}$; Leo Pharma) [12]. Culture medium was replaced twice a week, and the cells were detached with TrypLE Select CTS (Life Technologies). The cells were plated at a density of $1 \times$ $10^{3}$ cells $/ \mathrm{cm}^{2}$ from P1 onwards.

\section{Phenotype assessment}

Immunophenotypic characterization of BM-MSC was performed using the following antibodies. The Barcelona protocol used mouse anti-human CD45-fluorescein isothiocyanate (CD45-FITC, HI30, BD Pharmingen), anti-human CD105-phycoerythrin (CD105-PE, 43A4E1;
Miltenyi Biotec), anti-human HLA-DR-FITC (L243; BD Biosciences), anti-human CD90-PE (F15-42-1-5, Beckman Coulter), mouse anti-human CD31-FITC (WM59; BD Pharmingen), and mouse anti-human CD73-PE (AD2, BD Pharmingen). The Helsinki protocol used cell surface antigens CD44 (CD44 -FITC, G44-26; BD Pharmingen), CD49e (CD49e-PE, IIA1; BD Pharmingen), CD13 (CD13APC, WM15; BD Pharmingen), CD90 (CD90-FITC, 5E10; Stemcell Technologies and BD Pharmingen), CD73 (CD73-PE, AD2; BD Pharmingen), CD29 (CD29-APC, MAR4; BD Pharmingen), CD105 (CD105-PE, 266, BD Pharmingen; 43A3, Stem Cell Technologies; and MEM229, Abcam), CD14, (CD14-PE, M5E2; BD Pharmingen), CD19 (CD19-PE, HIB19; BD Pharmingen), CD34 (CD34PE, 8G12, BD Biosciences; AC136, Miltenyi Biotec), CD45 (CD45-PE, H130; BD Pharmingen), and HLA-DR (HLADR -PE, MEM-12, Abcam; L243, BD Biosciences).

Cells were stained for $15 \mathrm{~min}$ at room temperature (Barcelona) and for $30 \mathrm{~min}$ on ice (Helsinki), washed, and resuspended in phosphate-buffered saline (DPBS; Gibco). Non-specific cell staining was ruled out by using mouse immunoglobulin isotype controls (BD Pharmingen). The acquisition was performed on a FACSCalibur (Barcelona) or a Navios cytometer (Helsinki), and data were analyzed with the FlowJo software (TreeStar Inc., Ashland, OR, USA).

In both laboratories, HLA-DR expression was analyzed and used only for informative purposes but not for product release.

Re-analysis of HLA-DR for the standardization of data analysis from the two groups was performed using FlowJo. The gating strategy with the use of IgG isotype control for unspecific staining (below 1\%) is shown in Additional file 1: Figure S1.

\section{Differentiation assays}

Specific differentiation media were used for the osteogenic, chondrogenic, and adipogenic induction of undifferentiated MSC cultures in vitro as reported previously [12-14]. Safranin O (Sigma), Oil Red O (Sigma), Sudan III (Sigma), and von Kossa (Sigma) stainings were performed for the determination of the outcome of the differentiation assays.

\section{Cell count, viability, and apoptosis}

Cells were counted either by Trypan blue dye exclusion methods or with Perfect-Count Microspheres (Cytognos) in a FACSCalibur cytometer (Becton Dickinson). Viability was determined using the 7 -amino-actinomycin $\mathrm{D}$ (7-AAD, BD Biosciences) exclusion method and expressed as a percentage (\%) of total cells. Data were analyzed with the CellQuest Pro (Becton Dickinson) software. Alternatively, cell numbers and viability were determined using 
the NucleoCounter NC-100 device (ChemoMetec A/S, Denmark).

\section{Lymphocyte proliferation assays}

The immunomodulation potential of BM-MSC was tested in the presence of peripheral blood mononuclear cells (PBMC) at a ratio of 1:5 (Barcelona procedure) and 1:10, 1:20, and 1:50 (Helsinki procedure) as described elsewhere [12, 15]. The proliferation of carboxyfluorescein diacetate succinimidyl ester (CFSE)-labeled PBMC after polyclonal stimulation was determined by measuring the reduction of fluorescence intensity at day 5 by flow cytometry.

\section{Effect of different medium supplements}

The effect of different medium supplements on the variation of HLA-DR expression was investigated after incubation of the same BM-MSC lines with different batches of sera. For this purpose, three independent BM-MSC lines were expanded under the same conditions for one passage and subsequently incubated with either one of two different conditions for an additional passage: one condition that was previously found experimentally related to activation of BM-MSC $(14.3 \pm 12.8 \%$ HLA-DR ${ }^{+}$cells, $\left.n=7\right)$ and another one related to low HLA-DR ${ }^{+}$cell number in culture $\left(3.3 \pm 3.3 \%\right.$ HLA-DR ${ }^{+}$ cells, $n=2$ ).

\section{Activation of MSC}

For cell activation experiments, IFN- $\gamma$ (R\&D Systems, Minneapolis, MN, USA) was used as a stimulus at 10 , 100 , and $200 \mathrm{UI} / \mathrm{mL}$, based on the concentrations used in previously reported experiments [16].

For the recovery after stimulation experiments, the same line was used for all three conditions. Cells were previously stimulated $48 \mathrm{~h}$ with $100 \mathrm{UI} / \mathrm{mL}$ of IFN- $\gamma$. After $48 \mathrm{~h}$ incubation, cells were left without IFN- $\gamma$ to see the decrease in HLA-DR. Controls without stimulation at any point and with maintained stimulation with $100 \mathrm{UI} / \mathrm{mL}$ of IFN- $\gamma$ were performed.

\section{Data analysis}

Descriptive data were expressed as mean \pm standard deviation. Pearson correlation $p$ values were calculated to evaluate the significance of the correlation of cell density and HLA-DR expression in BM-MSC.

\section{Results}

\section{BM-MSC express variable levels of HLA-DR}

Despite compliance with the ISCT criteria, BM-MSC in culture showed variable percentages of HLA-DR ${ }^{+}$cells in 130 batches produced in two manufacturing sites (namely, Barcelona, $n=91$, and Helsinki, $n=39$ ). HLA-DR expression in Barcelona batches ranged from $<1$ to $77.7 \%$ (average $19.8 \pm 15.6 \%$, from patients enrolled in clinical trials for autologous use), whereas batches in Helsinki ranged from $<1$ to $60.5 \%$ (average $19.2 \pm 17.4 \%$, from healthy donors for allogeneic use) (Table 1). In order to bring consistency to the comparability of analyses performed in different laboratories following different techniques, we reanalyzed a fraction of data from both laboratories $(n=65$ and $n=37$ from Barcelona and Helsinki, respectively; Additional file 1: Figure S2) BMMSC batches from both centers complied with the specifications approved by their own respective regulatory authority in terms of phenotype, adherence to culturetreated plastic surfaces, fibroblastic morphology, differentiation potential, and immunomodulation capacity in vitro (Additional file 1: Table S1, Fig. 1).

In order to determine the potential triggers of cell activation in culture, standard parameters were analyzed, including density, medium supplements, and forced activation with IFN- $\gamma$ and its recovery after removal of the stimulus.

\section{Effect of cell density and passaging on HLA-DR expression} Regarding cell density and HLA-DR expression, no correlation was found (Fig. 2); dispersion without statistical correlation was observed in both cases ( $p$ value $=0.2287$ and 0.2272, in Barcelona and Helsinki datasets, respectively). However, culture passaging was found to change the HLA-DR ${ }^{+}$cell percentage. We collected HLA-DR values from the two passages required in the production process, which typically take a total of 3 weeks and correspond to $24.6 \pm 1.5$ cumulative population doublings (CPD). We observed a decrement in HLA-DR positivity of at least $25 \%$ in $71.9 \%$ of the cultures, whereas only $5.3 \%$ increased HLA-DR more than $25 \%$ of its initial value $(n=57)$. Furthermore, one MSC line was cultured up to passage 5 confirming the decrease in HLA-DR ${ }^{+}$ cell number (Additional file 1: Figure S3).

\section{Effect of cell culture medium supplement on HLA-DR expression}

Different batches of sera experimentally related with high and low HLA-DR values were tested in three BM-MSC lines derived from three different donors.

Table 1 Summary of HLA-DR expression in 130 batches of clinical-grade BM-MSC from two independent GMP-compliant facilities. Batches of BM-MSC were distributed according to the production site

\begin{tabular}{llcccc}
\hline Group & Number & Mean (\%) & $\begin{array}{l}\text { Standard } \\
\text { deviation } \\
(\%)\end{array}$ & $\begin{array}{l}\text { Minimum } \\
(\%)\end{array}$ & $\begin{array}{l}\text { Maximum } \\
(\%)\end{array}$ \\
\hline Barcelona & 91 & 19.8 & 15.6 & $<1$ & 77.7 \\
Helsinki & 39 & 19.2 & 17.4 & $<1$ & 60.5 \\
Total & 130 & & & & \\
\hline
\end{tabular}




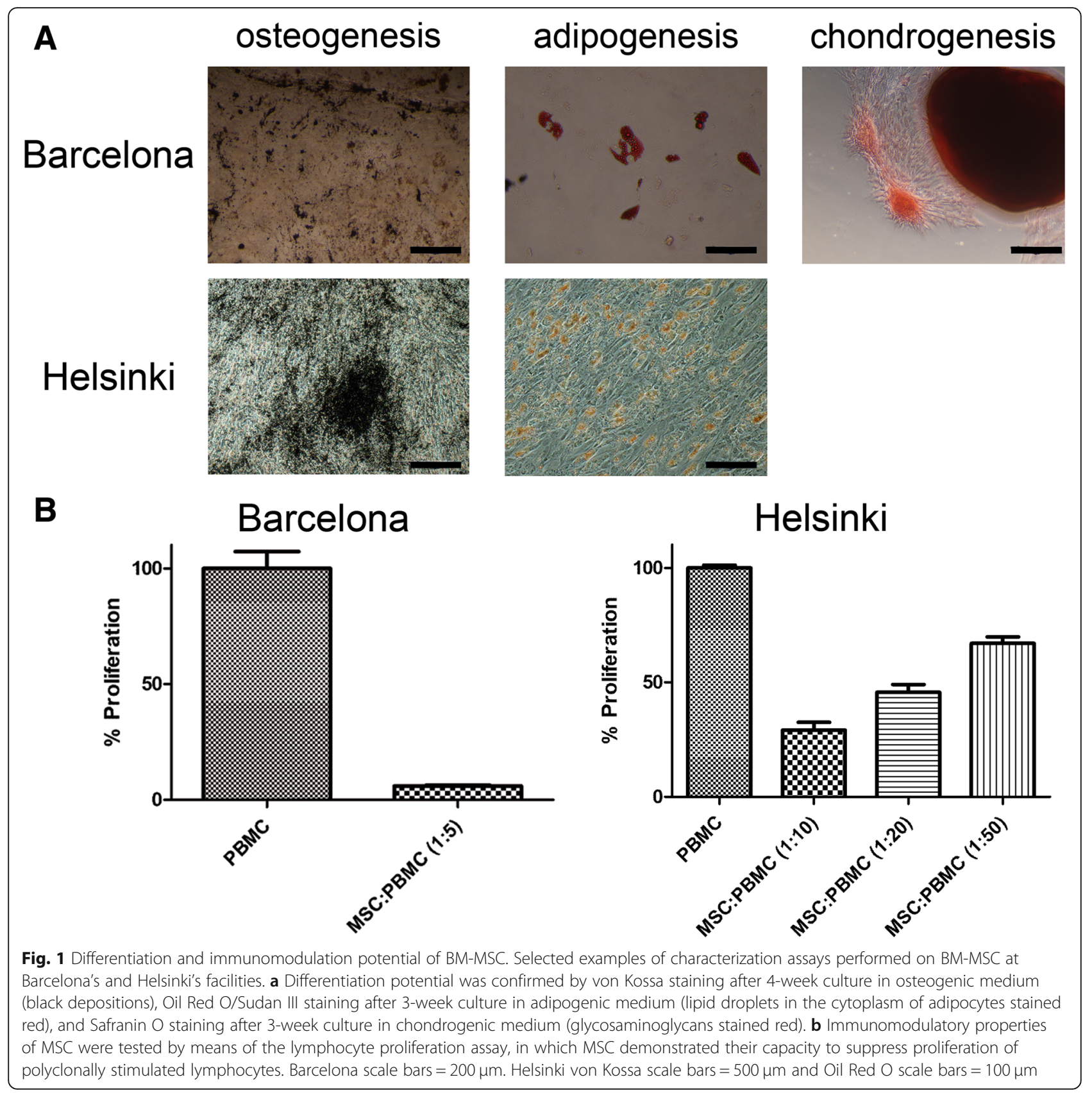

Despite starting with similar HLA-DR values (17.1\%, $19.4 \%$, and $15.0 \%$; corresponding to an average of $17.2 \% \pm 2.2 \%)$, the percentage of activation after incubation varied independently of the cell line or supplement. Remarkably, none of the other mesenchymal surface markers depended on HLA-DR expression (Table 2). Additionally, the potentiality of the three cell lines was not altered in either case, showing a similar capacity to differentiate in vitro into the osteogenic and chondrogenic lineages (Additional file 1: Figure S4).

\section{Activation of HLA-DR expression}

We took advantage of IFN- $\gamma$ being a strong activator of MSC in vitro and performed a series of experiments to study the effect of its addition to determine the kinetics of BM-MSC activation upon stimulation with increasing doses of IFN- $\gamma$. We observed that the maximum activation was reached at $48 \mathrm{~h}$ after induction, resulting in high levels of activation when using 100 and $200 \mathrm{UI} / \mathrm{mL}$ doses (Fig. 3a). Interestingly, when IFN- $\gamma$ stimulus was removed, the number of HLA-DR ${ }^{+}$cells decreased gradually over time (Fig. 3b). 

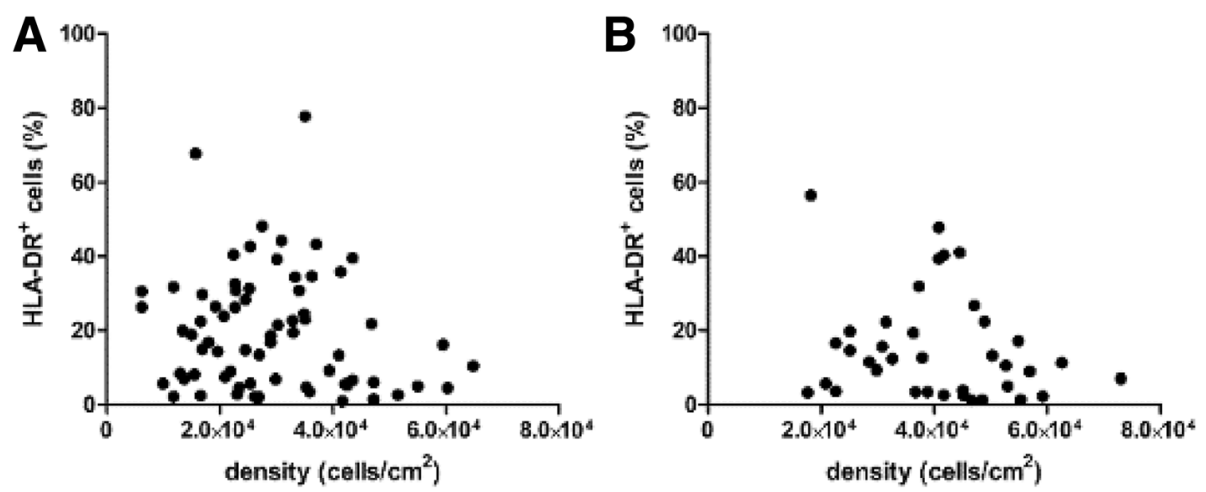

Fig. 2 Analysis of cell culture parameters. Effect of cell density on HLA-DR expression was studied in batches released from both manufacturing sites, namely Barcelona (a) and Helsinki (b). However, high dispersion without statistical correlation was observed in both cases $(p$ value $=0.2287$ and 0.2272 , respectively)

\section{Discussion}

Variability of HLA-DR expression in MSC cultures is a nuisance for developers adhering to the criteria established by the ISCT, so it tends to be overlooked provided that quality control $(\mathrm{QC})$ panels are adapted to robust product specifications in order to bring consistency from batch to batch using reliable markers [17]. This makes it difficult to fully realize how different cell preparations from different production sites can be. In this context, standardization of QC criteria and methodology is a pressing need as new MSC-based therapies reach the clinical setting and comparability of studies is required to define the efficacy of such new drug candidates $[4,5]$.

In the present study, we focused on the analysis of HLA class II molecule expression in cultured MSC which was demonstrated to be unpredictable and dynamic over time thus confirming previous studies [8]. Expression of HLA-DR has been proved, both in the present work and reported by others, that it can be triggered in vitro by IFN- $\gamma[9,18]$ and other pro-inflammatory stimuli [19]. Interestingly, when IFN- $\gamma$ stimulus was removed, the number of HLA-DR ${ }^{+}$cells gradually decreased over time. This observation is relevant since HLA-DR expression on cells during expansion cultures is variable over time; however, in-process and final product quality controls are performed only at specific time points during the production process, typically after 8 to 10 days from seeding, therefore showing only a snapshot of the status of the cells and as a result could be potentially masking a dynamic pattern of expression of HLA-DR. Regardless of HLA-DR, the identity of MSC is clearly defined by other parameters, such as the presence of mesenchymal cell surface markers and absence of hematopoietic and endothelial markers, fibroblastic cell morphology on plastic surfaces, and immunomodulation and differentiation potential [7]. Indeed, we also showed that HLA-DR expression had no effect on immunomodulation and multilineage differentiation potential of MSC [8].

In one of the first reports on this topic, Le Blanc and collaborators analyzed the intracellular protein levels of HLA-DR in MSC in parallel with extracellular HLA-DR phenotype by flow cytometry [18]. They found that MSC

Table 2 Result summary after expanding cells in different medium formulations associated with "activating" or "neutral" cues. Activation of cells occurred randomly regardless of the supplement used. Nonetheless, differentiation potential was not altered (please refer to Additional file 1: Figure S2)

\begin{tabular}{lllllllll}
\hline Cell line & Passage number & supplement & \%CD31 & \%CD45 & \%HLA-DR & \%CD73 & $\%$ CD105 & \%CD90 \\
\hline 1 & P0 & & 3.6 & 1.9 & 17.1 & 99.8 & 99.9 & 99.83 \\
& P1 & Activating & 2.0 & 0.3 & 14.2 & 99.7 & 99.9 & 99.6 \\
& P1 & Neutral & 7.7 & 2.0 & 29.6 & 99.3 & 99.4 & 99.4 \\
2 & & 6.4 & 1.1 & 19.4 & 100 & 98.7 & 99.9 \\
& P0 & Activating & 8.4 & 3.0 & 37.7 & 99.7 & 99.6 & 99.9 \\
& P1 & Neutral & 7.6 & 3.3 & 40.9 & 99.8 & 99.5 & 99.9 \\
& P1 & & 7.2 & 1.0 & 15.0 & 99.7 & 99.7 & 99.84 \\
& P0 & Activating & 4.7 & 3.3 & 23.9 & 99.4 & 99.4 & 99.5 \\
& P1 & Neutral & 4.3 & 2.9 & 25.4 & 99.1 & 99.3 & 99.3 \\
\hline
\end{tabular}

$P$ passage number 
HLA-DR expression in BM-MSC
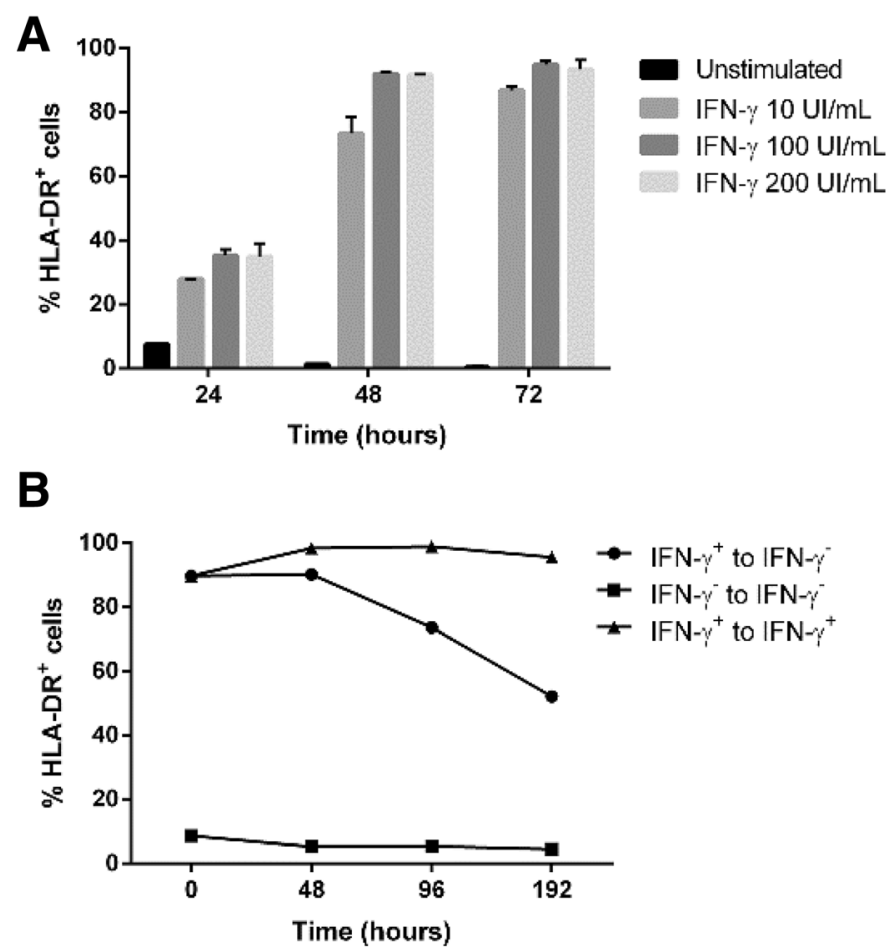

Fig. 3 Dynamics of BM-MSC activation in vitro. In culture, BM-MSC are activated by the addition of IFN- $\gamma$, which is illustrated by increasing concentration up to $200 \mathrm{UI} / \mathrm{mL}$, reaching a peak $48 \mathrm{~h}$ after stimulation (a). Interestingly, the reversal of the process for the recovery of HLA-DR expression levels using IFN- $\gamma$-activated cells takes longer than the activation time (b)

expressed first HLA-DR at the protein level intracellularly, and only later HLA-DR molecules were detected in the cellular membrane upon activation. The intracellular synthesis of HLA-DR was initiated after 2 days of exposure to IFN- $\gamma$, but required up to 7 days for cell surface expression. Nevertheless, we detected MSC activation extracellularly after $48 \mathrm{~h}$ after IFN- $\gamma$ exposure and provided data of slow HLA-DR downregulation. With this, it seems clear from these studies that the expression of HLA-DR can come from a previous exposure since clinical-grade manufacture bioprocesses are relatively short (that is, two passages only) and we provided evidence of variability of HLA-DR expression even in the very first passage. One might think that the condition of the donor could determine MSC activation in culture; however, our study collected data from both healthy donors and patients, and in both cases, the occurrence of HLA-DR expression appeared to be random.

With the removal of HLA-DR expression of MSC from the QC panels, and the acceptance that HLA-DR expression in cultured MSC does not follow a predictable pattern under standard in vitro growth conditions, it would be interesting to further investigate its triggers as its expression is known to be indicative of inflammatory microenvironments and maybe also differentiation commitment [20]. In any case, the major concern regarding HLA-DR expression in MSC would be the potential rejection of these cells. Lately, new hypotheses about the MSC mechanisms of action involved are being proposed, and among them, efferocytosis is gaining strength. This hypothesis supports that MSC releases soluble molecules and extracellular macrovesicles that signal to the target environment and then disappear [6]. Without considering HLADR expression, MSCs have not been reported to cause rejection, but on the contrary, MSCs are being used in the management of graft-versus-host disease (GvHD) [21, 22]. The mechanisms by which these cells induce tolerance were reported by Galleu and collaborators in vivo in an animal model and were apoptosis of MSC resulted in the induction of immunosuppression [23]. Interestingly, IFN- $\gamma$ activated MSCs are being used for the treatment of GvHD to induce tolerance [9]. Moreover, Zachar and collaborators illustrated that MSCs have to be activated in order to provide their therapeutic effects in the regenerative medicine field [19]. Therefore, the presence of HLA-DR molecules on the cell surface does not compromise MSC identity, 
neither their function and application in the clinical setting for immunomodulation and differentiation potential applications. Further clinical studies could discard the secondary effects of activated MSC as well as their therapeutic effect.

\section{Conclusions}

We concluded that the use of HLA-DR as a negative marker of BM-MSC does not add any additional value to QC panels and its expression does not affect other attributes, such as phenotype and functionality in vitro. Furthermore, we showed for the first time that the expression of HLA-DR is dynamic in MSC cultures. Finally, we believe that we can only reliably describe the full clinical potential of MSC if robust QC panels directly related to MSC's functionality are characterized, so the definition and broad adoption of meaningful potency assays are urgently needed.

\section{Additional file}

Additional file 1: Table S1. Release criteria of clinical-grade batches of BM-MSC from two independent GMP-compliant facilities. Release criteria defined on MSC phenotype. Figure S1. Gating strategy used in flow cytometry analysis to determine HLA-DR expression in BM-MSC. Represented in blue lgG isotype control for unspecific staining. The interval gate was established with a value of $\mathrm{HLA}^{-\mathrm{DR}^{+}}$of isotype control positivity below 1\%. Figure S2. Comparison of HLA-DR covariance between batch release and after standardization of the analysis of cytometric data from Barcelona and Helsinki. Statistically not significant ( $p$ value 0.2997). Figure S3. Percentage of MSC expressing HLA-DR along culture passaging. Figure S4. Osteogenic and chondrogenic potential of cells cultured in the presence of activating and non-activating sera supplements. Scale bars $=200 \mu \mathrm{m}$. (DOCX $16212 \mathrm{~kb})$

\section{Abbreviations}

BM: Bone marrow; CFSE: Carboxyfluorescein diacetate succinimidyl ester; CPD: Cumulative population doublings; FITC: Fluorescein isothiocyanate; GMP: Good Manufacturing Practice; HLA: Human leukocyte antigen; MSC: Multipotent mesenchymal stromal cells; P: Passage number; PBMC: Peripheral blood mononuclear cells; PE: Phycoerythrin; QC: Quality control

\section{Acknowledgements}

The authors would like to acknowledge the former members of Xcelia and current members of Servei de Teràpia Celllular (Banc de Sang i Teixits, Barcelona) for the technical support and advice and Drs. E. Cantó and F. Rudilla for critically reviewing the manuscript.

\section{Authors' contributions}

MG-V and AL performed the experiments, analyzed the data, and wrote the manuscript. JN and JV conceived the study, revised the data and, wrote the manuscript. All authors approved the final version of the manuscript.

\section{Funding}

BST is a member of the Spanish Cell Therapy Network (Red de Terapia Celular, TerCel, expedient No. RD16/0011/0028), awarded by the Generalitat de Catalunya as Consolidated Research Group (ref. 2017SGR719) and developed in the context of AdvanceCat with the support of ACCIÓ (Catalonia Trade \& Investment; Generalitat de Catalunya) under the Catalonian ERDF operational program (European Regional Development Fund) 2014-2020.

\section{Availability of data and materials}

All datasets generated for this study are included in the manuscript and the supplementary files.

\section{Ethics approval and consent to participate}

Cells were sourced from Banc de Sang i Teixits' Biobank (Barcelona, Spain) and had appropriate donor informed consent for use in research. An authorization is issued by Hospital de la Vall d'Hebron's Ethics Committee (Barcelona, Spain) to JV.

\section{Consent for publication}

Not applicable.

\section{Competing interests}

The authors declare that they have no competing interests.

\section{Author details}

${ }^{1}$ Servei de Teràpia Cel.lular, Banc de Sang i Teixits, Edifici Dr. Frederic Duran i Jordà, Passeig Taulat, 116, 08005 Barcelona, Spain. ${ }^{2}$ Transfusion Medicine Group, Vall d'Hebron Research Institute (VHIR), Universitat Autònoma de Barcelona, Passeig de la Vall d'Hebron 129-139, 08035 Barcelona, Spain. ${ }^{3}$ Finnish Red Cross Blood Service, Advanced Cell Therapy Centre, Kivihaantie 7, FIN-00310 Helsinki, Finland. " Musculoskeletal Tissue Engineering Group, Vall d'Hebron Research Institute (VHIR), Universitat Autònoma de Barcelona, Passeig de la Vall d'Hebron 129-139, 08035 Barcelona, Spain. 'Departament de Medicina, Universitat Autònoma de Barcelona, Passeig de la Vall d'Hebron 129-139, 08035 Barcelona, Spain.

Received: 5 April 2019 Revised: 13 May 2019 Accepted: 23 May 2019 Published online: 13 June 2019

\section{References}

1. Mohal JS, Tailor HD, Khan WS. Sources of adult mesenchymal stem cells and their applicability for musculoskeletal applications. Curr Stem Cell Res Ther. 2012;7(2):103-9.

2. Murphy MB, Moncivais K, Caplan Al. Mesenchymal stem cells: environmentally responsive therapeutics for regenerative medicine. Exp Mol Med. 2013:45:e54.

3. Guadix JA, Zugaza JL, Galvez-Martin P. Characteristics, applications and prospects of mesenchymal stem cells in cell therapy. Med Clin (Barc). 2017;148(9):408-14.

4. Vives J, Mirabel C. Multipotent mesenchymal stromal cells from bone marrow for current and potential clinical applications. Reference module in biomedical sciences: Elsevier; 2018. https://doi.org/10.1016/B978-0-12801238-3.65506-X, https://www.sciencedirect.com/science/article/pii/ B978012801238365506X.

5. Cuende N, Rasko JEJ, Koh MBC, Dominici M, Ikonomou L. Cell, tissue and gene products with marketing authorization in 2018 worldwide. Cytotherapy. 2018;20(11):1401-13.

6. Galipeau J, Sensebe L. Mesenchymal stromal cells: clinical challenges and therapeutic opportunities. Cell Stem Cell. 2018:22(6):824-33.

7. Dominici M, Le Blanc K, Mueller I, Slaper-Cortenbach I, Marini F, Krause D, et al. Minimal criteria for defining multipotent mesenchymal stromal cells. The International Society for Cellular Therapy position statement. Cytotherapy. 2006;8(4):315-7.

8. Grau-Vorster M, Rodriguez L, Torrents-Zapata S, Vivas D, Codinach M, Blanco M, et al. Levels of IL-17F and IL-33 correlate with HLA-DR activation in clinical-grade human bone marrow-derived multipotent mesenchymal stromal cell expansion cultures. Cytotherapy. 2019;21(1):32-40.

9. Polchert D, Sobinsky J, Douglas G, Kidd M, Moadsiri A, Reina E, et al. IFNgamma activation of mesenchymal stem cells for treatment and prevention of graft versus host disease. Eur J Immunol. 2008;38(6):1745-55.

10. Codinach M, Blanco M, Ortega I, Lloret M, Reales L, Coca MI, et al. Design and validation of a consistent and reproducible manufacture process for the production of clinical-grade bone marrow-derived multipotent mesenchymal stromal cells. Cytotherapy. 2016;18(9):1197-208.

11. Salmenniemi $U$, Itälä-Remes M, Nystedt J, Putkonen M, Niittyvuopio R, Vettenranta K, et al. Good responses but high TRM in adult patients after MSC therapy for GvHD. Bone Marrow Transplant. 2017;52(4):606-8.

12. Laitinen A, Oja S, Kilpinen L, Kaartinen T, Möller J, Laitinen S, et al. A robust and reproducible animal serum-free culture method for clinical-grade bone marrowderived mesenchymal stromal cells. Cytotechnology. 2016;68(4):891-906.

13. Caminal M, Velez R, Rabanal RM, Vivas D, Batlle-Morera L, Aguirre M, et al. A reproducible method for the isolation and expansion of ovine 
mesenchymal stromal cells from bone marrow for use in regenerative medicine preclinical studies. J Tissue Eng Regen Med. 2017;11(12):3408-16.

14. Vivas D, Caminal M, Oliver-Vila I, Vives J. Derivation of multipotent mesenchymal stromal cells from ovine bone marrow. Curr Protoc Stem Cell Biol. 2018;44:2B 9 1-2B 922.

15. Oliver-Vila I, Ramirez-Moncayo C, Grau-Vorster M, Marin-Gallen S, Caminal M, Vives J. Optimisation of a potency assay for the assessment of immunomodulative potential of clinical grade multipotent mesenchymal stromal cells. Cytotechnology. 2018;70(1):31-44.

16. Tang KC, Trzaska KA, Smirnov SV, Kotenko SV, Schwander SK, Ellner JJ, et al. Down-regulation of MHC II in mesenchymal stem cells at high IFN-gamma can be partly explained by cytoplasmic retention of CIITA. J Immunol. 2008;180(3):1826-33.

17. Mendicino M, Bailey AM, Wonnacott K, Puri RK, Bauer SR. MSC-based product characterization for clinical trials: an FDA perspective. Cell Stem Cell. 2014;14(2):141-5.

18. Le Blanc K, Tammik C, Rosendahl K, Zetterberg E, Ringden O. HLA expression and immunologic properties of differentiated and undifferentiated mesenchymal stem cells. Exp Hematol. 2003;31(10):890-6.

19. Zachar L, Bacenkova D, Rosocha J. Activation, homing, and role of the mesenchymal stem cells in the inflammatory environment. J Inflamm Res. 2016:9:231-40

20. Reyes-Botella C, Montes MJ, Vallecillo-Capilla MF, Olivares EG, Ruiz C. Expression of molecules involved in antigen presentation and $\mathrm{T}$ cell activation (HLA-DR, CD80, CD86, CD44 and CD54) by cultured human osteoblasts. J Periodontol. 2000;71(4):614-7.

21. Ryan JM, Barry FP, Murphy JM, Mahon BP. Mesenchymal stem cells avoid allogeneic rejection. J Inflamm (Lond). 2005;2:8.

22. English K, Wood KJ. Mesenchymal stromal cells in transplantation rejection and tolerance. Cold Spring Harb Perspect Med. 2013;3(5):a015560.

23. Galleu A, Riffo-Vasquez Y, Trento C, Lomas C, Dolcetti L, Cheung TS, et al. Apoptosis in mesenchymal stromal cells induces in vivo recipient-mediated immunomodulation. Sci Transl Med. 2017;9(416). https://doi.org/10.1126/ scitransImed.aam7828, https://stm.sciencemag.org/content/9/416/ eaam7828.short.

\section{Publisher's Note}

Springer Nature remains neutral with regard to jurisdictional claims in published maps and institutional affiliations.

Ready to submit your research? Choose BMC and benefit from:

- fast, convenient online submission

- thorough peer review by experienced researchers in your field

- rapid publication on acceptance

- support for research data, including large and complex data types

- gold Open Access which fosters wider collaboration and increased citations

- maximum visibility for your research: over $100 \mathrm{M}$ website views per year

At $\mathrm{BMC}$, research is always in progress.

Learn more biomedcentral.com/submissions 